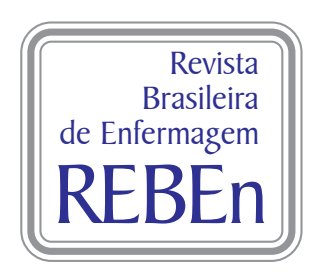

\title{
Cuidados cotidianos
}

\author{
Daily care \\ Cuidados cotidianos
}

\begin{abstract}
Maria José Coelho'
'Universidade Federal do Rio de Janeiro. Escola de Enfermagem Anna Nery. Departamento de Enfermagem Médico-Cirúrgica. Rio de Janeiro, RJ
\end{abstract}

Submissão: 19/08/2008

Aprovação: 10/07/2010

\section{RESUMO}

Objetivo: Apresentar via Quadros os cuidados de enfermagem de 15.204 clientes adultos enfermos e hospitalizados com doenças crônicas ou agudas. Método: Pesquisa Quantitativa realizada em um hospital de 527 leitos, e coleta de dados via relatório diário de enfermagem. Resultados: Movimentação dos cuidados; Condições dos clientes; e Situações dos Clientes, emergiu os cuidados desde o momento da entrada até a saída Hospitalar. Conclusão: A recuperação do cliente não se esgota nas possibilidades de atenção e equilíbrio dos males orgânicos previstos pela cura, calcados no modelo Que identifica a cura e a patologia como principais referenciais. A alta hospitalar demonstra o impacto/resultado dos cuidados recebidos na sua recuperação e uma rede intricada numa cadeia continua e diária desses três eixos.

Descritores: Cuidado; Enfermagem; PesQuisa em enfermagem.

\section{ABSTRACT}

Purpose: To present via charts, the nursing care of 15.204 hospitalized adult clients with chronic or severe diseases. Methodo: Quantitative research carried out in a 527-bed hospital, with data collection using the nursing notes. Results: Care movement; client conditions; client situations; the care that took place was one from the moment the client checked in to the moment he checked out of the hospital. Conclusion: The data consolidate what observed in the Hospital Routine: that the recovery of the client is not over within the possibilities of attention and balance of the organic ills predicted by the cure of these subjects, based on the model which identifies the cure as well as the pathology as the main references. From the total number of patients, a great number were able to leave the hospital, which shows the impact/result of the care received during their recovery, and a complex network in a continuous and daily chain in those three axis.

Key words: Care; Nursing; Nursing research.

\section{RESUMEN}

Objetivos: Presentar através de unos cuadros los cuidados de enfermería de 15.204 clientes adultos yhospitalizados con enfermedades crónicas o agudas. Métodos: Investigación cuantitativo realizada en uno hospital con 527 camas, con colecta de los datos via relatos del diario de enfermería aplicado diariamente. Resultados: Movilizacón cuidados; Condiciones de los clientes y; Situaciones de los clientes presentados en cuadros, fueron realiados los cuidados desde el momento de la entrada en el sistema de hospitalización hasta la salida. Conclusión: La recuperación del cliente no se agota en las posibilidades de la atención y equilibrio de los males orgánicos previstos por la cura de estos sujeitos calcados en el modelo Que identifica la cura y a patologia como principales referencias, un alto número fue dado de alta hospitalización, lo que demuestra Que el impacto/resultado de los cuidados en una red intricada de una cadena continua y diaria de esos tres ejes.

Descriprotes: Cuidado; Enfermería; Investigación en enfermería.

\footnotetext{
AUTOR CORRESPONDENTE Maria José Coelho. Escola de Enfermagem Anna Nery. Rua Afonso Cavalcanti, 275. Cidade Nova. CEP 202 I I-I I0. Rio de Janeiro, RJ. E-mail: zezecoelho@yahoo.com.br
} 


\section{INTRODUÇÃO}

Trata o presente dos cuidados cotidianos de enfermagem prestados diariamente no ano de 2006 num entrelaçamento da Ciência do Cuidado com a Ciência do Cotidiano. O cuidar e os cuidados recebidos pelos clientes adultos hospitalizados e reinternados com doenças crônicas e agudas são compostos da Arte de organizar o próprio pensamento, de (re)ligar e ao mesmo tempo diferenciar, de contextualizar e globalizar, de relacionar cada informação e cada conhecimento ao seu contexto e conjunto saberes particulares ao conhecimento global por outro lado, exige do cliente uma resposta orgânicapsicosocial imediata e tardia aos cuidados recebidos expressos como resposta aos cuidados prestados. Os Objetivos dessa pesquisa foram apresentar os cuidados de enfermagem ao cliente adulto enfermo e hospitalizado com doenças crônicas ou agudas e gerar conhecimentos de Enfermagem que ecoam do cotidiano de forma silenciosa.

\section{MÉTODO}

Foram utilizado os conceitos de cotidiano de Certeau, Michael (1) "extrair do seu ruído as maneiras de fazer; os de Coelho, Figueiredo e Carvalho ${ }^{(2)}$ de cuidar como processo de expressão, de reflexão, de elaboração do pensamento, de imaginação, de meditação e de aplicação intelectual [...]"; cuidados "ação imediata prestada [...], em curto espaço de tempo, desenvolvido em vários momentos, envolvendo segurança e competência, aliadas à tecnologia especifica que a situação exige" e cliente "é aquele que sofreu uma violência ou acidente físico e/ou orgânico, e Que traz consigo as suas características pessoais, familiares e sociais, necessitando urgentemente, do cuidar/cuidados de enfermagem[...]".

Optamos, para apresentar os cuidados diários através de uma abordagem Quantitativa descritiva e exploratória desenvolvida durante as reuniões semanais no Laboratório de Tecnologia e Procedimentos de Enfermagem EEAN/CCS/UFRJ/Brasil com estabelecimentos de relações na busca do cuidar e cuidados no ano de 2006 (janeiro a dezembro), totalizando 15204 clientes homens e mulheres, adultos, internados e re-internados com doenças crônicas e agudas, cuidados, incluindo diferentes momentos, de circunstâncias práticas, descritíveis, inteligíveis, relatáveis e analisáveis do cotidiano em Enfermagem hospitalar pública e privada do Rio de Janeiro / Brasil.

O Hospital, neste estudo, é considerado laboratório de pesQuisa Que produz conhecimentos e saberes a cerca dos cuidados. O instrumento de coleta de dados foi o relatório 'Diário de Enfermagem do HUCFF/UFRJ', aplicado diariamente pelos enfermeiros plantonistas. A Resolução 196/96 foi atendida, nos seus artigos, e a pesquisa aprovada pelo Comitê de Ética no CEP 0095.0.197.197-06 de 05/09/2006 - CNS/MS - Comitê de Ética do HUCFF/UFRJ. A análise foi feita pelo programa Deplhi e o banco de dados cachê com o auxilio de 02 analistas de sistemas.

\section{RESULTADOS}

Demonstração dos cuidados cotidianos

No cotidiano Assistencial de Cuidar emergiu uma síntese das maneiras de cuidar dos clientes desde o momento da sua entrada no sistema hospitalar até a sua saída por alta, óbito ou transferência, com Quadros. O fluxograma ${ }^{(2)}$ do Cuidar e dos cuidados descreve o caminho que o cliente/cuidados percorre diariamente Quando internado. O cliente se registra e é admitido no Serviço de Admissão ou Setor ou Enfermaria - Recebido no Setor de Internação - Coleta de dados para o histórico de Enfermagem - Implementação dos primeiros cuidados hospitalares - atendimento médico - vigilância nos cuidados de Enfermagem de compartilhamento Implementações de Cuidados de Enfermagem de baixa complexidade - Complexos e de altíssima complexidade - Cuidar individual ou coletivo - Requisição de exames - resultados - encaminhamentos instituição de tratamento e consequente cuidados referentes a estes - Implementação - criação - improvisação de cuidados - A luta no limiar entre a vida e a morte - Cuidados na fronteira do limite. Em vigia e vigilância intensivos e sequencial dos cuidados de Enfermagem - Cuidar no Encaminhamento para o CTI - Centro Cirúrgico ou Setor de Internação - Cuidar no momento da morte óbito - Cuidar do corpo morto - Encaminhar o corpo morto à patologia - cuidar no tocante às providências legais Quanto ao corpo encaminhado ao Instituto médico legal, se for o caso - Cuidar da Família/amigos/companheiros/conhecidos, entre outros Que traduzem os cuidados de Enfermagem recebidos em uma multiplicidade de situações.

Os cuidados de enfermagem desse cotidiano Que percorrem esse caminho são frequentes e visíveis e as maneiras de cuidar em enfermagem ${ }^{(3)}$ são instrumentalizadas em três eixos: Movimentação do Cuidar (cuidar diurno e cuidar noturno, número de clientes, admissões, transferência, altas, liberações, óbitos e licença hospitalar); Condições dos clientes (independentes dos cuidados, dependência parcial e total dos cuidados, conscientes, desorientados, inconscientes e clientes psiQuiátricos internados por distúrbios orgânicos em hospital geral); Situações dos Clientes (acesso venoso periférico e profundo, número de medicações endovenosas e endovenosas especiais, glicemia capilar, NPT, QT, PVC, dieta enteral. SNG, vesical, drenos, ostomias, controle hídrico, preparo de exames, curativos, pré-operatórios, cirurgia de emergência, lesões de pele, precauções por contato e próteses respiratórias).

\section{Movimentação do Cuidar}

A movimentação do Cuidar e dos cuidados inclui os cuidar diurno (SD) e cuidar noturno (SN), $\mathrm{n}^{\circ}$ de clientes, admissões, transferência, altas, liberações, óbitos, licença hospitalar e Diária e Taxa de Ocupação Hospitalar. Nesse aspecto é importante destacar o Que Coelho ${ }^{(2-3)}$ descreve como Cuidar diurno e cuidar Noturno:

O Cuidar Diurno tem em seu núcleo o planejamento e execução dos cuidados de enfermagem durante o dia e requer uma organização especial, principalmente no período da manhã: movimentação de pessoas, num ambiente de entra e sai de clientes, familiares, enfermeiros e suas equipes, médicos, fisioterapeutas nutricionistas, professores, pesquisadores alunos de todos os níveis, ruídos de monitores, telefone que soa, exames, curativos, entre tantos outros.

lá o cuidar noturno trás o silêncio prolongado, modificações nos ritmos biológicos em relação ao Ciclo vigília/sono. É o relógio biológico, muitas vezes, em descompasso orgânico. Esse cuidar requer autonomia, processo decisório e tomado de decisão ${ }^{(2)}$. Tanto 
o Cuidar diurno Quanto o noturno trazem uma gama de particularidades em seus núcleos assim como nos cenários onde são desenvolvidos dentro de um modelo biologista das especialidades médicas Que são nominados os setores/enfermarias de Internação descritos a seguir.

Destaque se faz para as complexidades Que alguns cenários exigem em relação a outros na elaboração dos cuidados cotidianos prestados.

Mesmo na Questão leitos/dia, assim como para as admissões, chamam atenção os picos máximos e os totais apresentados na movimentação desses cuidados. De 181.999 leitos/dias as admissões variaram de 04 a 4.184 clientes mensais, movimentando um infinito número de cuidados prestados para atender as necessidades de acolhimento desses ao adentrar em um ambiente desconhecido que é uma unidade Hospitalar.

As altas variaram de zero a 3.981 clientes em um mês. Os óbitos foram de zero a 118 em um mês. Tanto para as altas Quantos para os óbitos os cuidados são de especificidades impares com

\begin{tabular}{|l|l|}
\hline \multicolumn{2}{|c|}{ Cenários dos cuidados } \\
\hline $\begin{array}{l}\text { Dermatologia Nefrologia } \\
\text { Unidade Cirurgia Cardíaca } \\
\text { Unidade Coronariana }\end{array}$ & Plástica, oftalmologia, otorrino, cirurgia pediátrica e ICCP \\
\hline Doença infecto-parasitaria & $\begin{array}{l}\text { Neurocirúrgia, Neurologia, } \\
\text { Urologia, Ginecologia }\end{array}$ \\
\hline Cardiologia, Hematologia Clínica & $\begin{array}{l}\text { Isolamento, Transplante de Medula, } \\
\text { Ortopedia }\end{array}$ \\
\hline Gastrologia, Clinica médica & Cirurgia geral \\
\hline Emergência & CTI-Cirúrgico \\
\hline Cirurgia vascular & CTI-Clínico \\
\hline Cirurgia cardíaca & CTI-Convênio \\
\hline Reumatologia, Endocrinologia, Proctologia & Enfermaria de cuidados intermediários \\
\hline
\end{tabular}

Quadro 1. Cenários dos Cuidados Cotidianos para os 527 leitos e 15.204 clientes.

\begin{tabular}{|ccc|}
\hline Leitos-Dia & Admissões & Total \\
\hline 5.840 & 249 & 256 \\
11.315 & 388 & 429 \\
13.870 & 545 & 873 \\
7.524 & 330 & 373 \\
12.045 & 619 & 748 \\
12.775 & 676 & 819 \\
6.205 & 615 & 728 \\
10.440 & 563 & 664 \\
21.987 & 4.184 & 4.251 \\
13.870 & 924 & 1.103 \\
12.775 & 670 & 781 \\
9.490 & 409 & 496 \\
15.695 & 1.219 & 1.412 \\
2.896 & 71 & 407 \\
2.172 & 40 & 187 \\
464 & 4 & 31 \\
1.340 & 21 & 116 \\
3.640 & 266 & 303 \\
10.220 & 313 & 391 \\
136 & 28 & 28 \\
2.920 & 138 & 156 \\
2.190 & 35 & 261 \\
2.190 & 209 & 391 \\
\hline 181.999 & 12.516 & 15.204 \\
\hline & & \\
\hline
\end{tabular}

Quadro 2. Movimentação referente a ocupação dos leitos/dia e dos Cuidados de Recebimento para admissão nas Unidades. 
entrelaçamento das ciências biológicas e humanas. Os Quadros 4,5 e 6 apresentam os números de leitos-dia, entradas e saídas assim como os números de diárias e taxa de ocupação onde os cuidados de enfermagem prestados se movimentam em direção a recuperação da saúde dos clientes hospitalizados.

A taxa de ocupação de $67,1 \%$ apresenta como os cuidados de enfermagem se mostram nessa mesma proporção ao serem prestados. Por outro lado, uma taxa de Quase $68 \%$ requer cuidados integrais e individuais respeitando a integralidade e individualidade de cada cliente hospitalizado.

\section{Condições dos clientes Que recebem os cuidados}

A condição dos clientes inclui as seguintes situações: independentes dos cuidados, dependência parcial e total dos cuidados; clientes conscientes, desorientados, inconscientes e clientes psieuiátricos internados por distúrbios orgânicos em hospital geral.

Outros dados importantes correlacionados com as condições dos clientes e a implementação e manutenção dos cuidados cotidianos diz respeito as taxas diárias de internação. A taxa de ocupação variou de $52,97 \%$ a $101,97 \%$, com média de 1,71 a 29,67. O índice de substituição dos leitos -ISS variou de $-0,32$ a 25,67. Já a taxa média de mortalidade hospitalar foi de zero a 27,68 e a taxa de mortalidade Institucional foi de 0,07 a 26,79 .

\section{Situações dos Clientes}

Os dados apresentados a seguir têm relação direta com a Situação Clinica dos Clientes hospitalizados e a necessidade dos cuidados de Enfermagem de acesso venoso periférico e profundo, $\mathrm{n}^{\mathrm{o}}$ de medicações endovenosas e endovenosas especiais, glicemia capilar, NPT, QT, PVC, dieta enteral. SNG, vesical, drenos, ostomias, controle hídrico, preparo de exames, curativos, pré-operatórios,

\begin{tabular}{|c|c|c|c|}
\hline T/I (Saída) & Alta & Óbito & Total \\
\hline 15 & 237 & 3 & 255 \\
37 & 372 & 19 & 428 \\
240 & 609 & 19 & 868 \\
75 & 288 & 17 & 380 \\
120 & 546 & 79 & 745 \\
137 & 555 & 118 & 810 \\
92 & 631 & 4 & 727 \\
86 & 571 & 7 & 664 \\
265 & 3981 & 3 & 4249 \\
135 & 945 & 25 & 1105 \\
113 & 674 & 12 & 799 \\
68 & 395 & 29 & 492 \\
180 & 1202 & 35 & 1417 \\
343 & 11 & 47 & 401 \\
122 & 13 & 49 & 184 \\
22 & 0 & 7 & 29 \\
78 & 3 & 31 & 112 \\
20 & 260 & 19 & 299 \\
33 & 339 & 17 & 389 \\
1 & 2 & 0 & 3 \\
22 & 119 & 16 & 157 \\
224 & 32 & 7 & 263 \\
245 & 123 & 586 & 12.494 \\
\hline 2.673 & 11.908 & & 391 \\
\hline & & 23 & \\
\hline
\end{tabular}

Quadro 3. Os Cuidados de Saídas por alta ou óbito.

\begin{tabular}{|c|c|c|c|}
\hline Leitos-Dia & T/I (Ent) & Admissões & Total \\
\hline 181.999 & 2.688 & 12.516 & 15.204 \\
\hline
\end{tabular}

Quadro 4. Panorama das entradas de clientes e leitos-dia.

\begin{tabular}{|c|c|c|c|}
\hline T/I (Saída) & Alta & Óbito & Total \\
\hline 2.673 & 11.908 & 586 & 12.494 \\
\hline
\end{tabular}

Quadro 5. Saídas por alta e óbitos. 


\begin{tabular}{|c|c|}
\hline Diárias & Taxa de Ocupação Hospitalar \\
\hline 121.963 & 67,01 \\
\hline
\end{tabular}

Quadro 6. Diárias e Taxa de Ocupação Hospitalar por onde circulam os cuidados.

\begin{tabular}{|c|c|c|c|}
\hline Sem Dependência & Dep.Parcial & Dep.Total & Plantão \\
\hline 50.211 & 37.703 & 25.523 & SN \\
\hline 50.364 & 36.029 & 26.680 & SD \\
\hline
\end{tabular}

Quadro 7. Nível de dependência dos cuidados de Enfermagem.

\begin{tabular}{|c|c|c|c|c|}
\hline Orientado & Desorientado & Inconsciente & PsiQuiatrico & Plantão \\
\hline 104.352 & 5.382 & 3.858 & 699 & SD \\
\hline 104.352 & 5.382 & 3.858 & 699 & SN \\
\hline
\end{tabular}

Quadro 8. Nível de consciência que demandam cuidados.

\begin{tabular}{|c|c|c|c|c|c|}
\hline \multicolumn{2}{|c|}{ Acesso venoso } & \multirow{2}{*}{ EV.Especial } & \multirow{2}{*}{ Glicemia.Capilar } & NPT & \multirow{2}{*}{ Plantão } \\
\cline { 1 - 2 } Periférico & AV.Profundo & & & & \\
\hline 39.183 & 15.947 & 27.244 & 35.355 & 1.916 & SD \\
\hline 38.407 & 16.221 & 27.799 & 35.206 & 1.848 & SN \\
\hline
\end{tabular}

Quadro 9. Cuidados Quanto à punção venosa.

\begin{tabular}{|c|c|c|c|c|}
\hline QT & PVC & Dieta Enteral & SNG Gast. & Plantão \\
\hline 1.445 & 3.614 & 6.828 & 10.058 & SD \\
\hline 1.000 & 4.191 & 6.895 & 10.330 & SN \\
\hline
\end{tabular}

Quadro 10. Cuidados Quanto à Quimioterapia, pressão venosa central, dieta enteral e sondagem nasogástrica.

cirurgia de emergência, lesões de pele, precauções por contato e próteses respiratórias.

Além dos cuidados listados, são envolvidos e não apresentados nos três eixos os cuidaods ${ }^{(3)}$ solidários, do ambiente, de promoção da saúde, de engajamento na sociedade, providenciar material e recursos humanos, equilibrar, resgatar os aspectos que se encontram encobertos pela hospitalização, passar visita diariamente, lidar com as prioridades, de se colocar no lugar do outro, chamar pelo nome próprio, respeitar a individualidade, se apresentar como enfermeiro, explicar os cuidados de enfermagem, olhar de forma holística, pensar e construir o cuidado, ouvir, assistir, orientar, diagnosticar, interagir, confortar, ter amor, ter fé, higienizar o corpo, registrar, admiti-lo, compartilhar com os outros profissionais de saúde, cuidar para saída/alta, para os exames complementares entre tantos outros 107 cuidados.

\section{CONCLUSÃO}

A apreensão dos cuidados de Enfermagem no cotidiano Assistencial para 15.204 clientes em doze meses imortaliza e consolida momentos, muitas vezes, (in)visíveis ${ }^{(4)}$ do cuidado humano e permiti saber mais sobre ele e apreender momentos distintos como resposta as situações emergentes e re-surgentes do processo saúde-doença. Quando o cliente recebe os cuidados, ele os recebe e reage ao mesmo de forma perceptível, também há uma mestiçagem de saberes tecidos a partir de contribuições universais, representações do espaço, do tempo e dos objetos na construção dos cuidados prestados. Esses conhecimentos vêm da mestiçagem de contribuições da física, Química, sociologia, antropologia, entre outras, e de contradições da vida cotidiana.

Os dados consolidam o Que observamos no Cotidiano Assistencial, Que a recuperação do cliente não se esgota nas possibilidades de atenção e equilíbrio dos males orgânicos, sociais e psicológicos previstos pela cura desses sujeitos calcados no modelo cartesiano mecanicista que identifica a cura e a patologia como principais referenciais.

O cuidado também é um referencial de recuperação organicopsicosocial do Qual há uma resposta visível dado pelo cliente enfermo hospitalizado com doença crônica ou aguda. Do total estudado, 15.204, Que ocuparam os 527 leitos no ano de 2006, e dos Quais 586 foram a óbito, pode-se fazer a seguinte correlação: a Taxa de ocupação Hospitalar foi de $67,01 \%$ e os Cuidados totalizaram 2.191.771, o Que mais uma vez demonstra o impacto/resultados dos cuidados recebidos na recuperação do Quadro clinico dos 


\begin{tabular}{|ccccccc|}
\hline Diárias & TOH & MPe & IIS & IR & TMGH & TMIn \\
\hline 3.643 & 62,38 & 14,29 & 8,62 & 15,89 & 1,18 & 1,18 \\
7.702 & 68,07 & 18,00 & 8,44 & 13,77 & 4,44 & 4,21 \\
10.058 & 72,52 & 11,59 & 4,39 & 22,78 & 2,19 & 1,61 \\
5.664 & 75,28 & 14,91 & 4,89 & 18,38 & 4,47 & 4,21 \\
12.282 & 101,97 & 16,49 & $-0,32$ & 22,51 & 10,60 & 10,47 \\
12.224 & 95,69 & 15,09 & 0,68 & 23,08 & 14,57 & 14,44 \\
3.581 & 57,71 & 4,93 & 3,61 & 42,65 & 0,55 & 0,55 \\
5.397 & 51,70 & 8,13 & 7,59 & 23,15 & 1,05 & 0,45 \\
7.250 & 32,97 & 1,71 & 3,47 & 70,34 & 0,07 & 0,07 \\
8.183 & 59,00 & 7,41 & 5,15 & 29,00 & 2,26 & 1,81 \\
9.294 & 72,75 & 11,63 & 4,36 & 22,77 & 1,50 & 1,38 \\
6.504 & 68,54 & 13,22 & 6,07 & 18,87 & 5,89 & 5,49 \\
10.223 & 65,14 & 7,21 & 3,86 & 32,86 & 2,47 & 2,19 \\
2.249 & 77,66 & 5,61 & 1,61 & 50,40 & 11,72 & 10,22 \\
2.006 & 92,36 & 10,9 & 0,90 & 30,84 & 26,63 & 26,63 \\
245 & 52,80 & 8,45 & 7,55 & 22,75 & 24,14 & 24,14 \\
1.190 & 88,81 & 10,63 & 1,34 & 30,42 & 27,68 & 26,79 \\
2.732 & 75,05 & 9,14 & 3,04 & 29,90 & 6,35 & 5,69 \\
5.778 & 56,54 & 14,85 & 11,42 & 13,85 & 4,37 & 3,86 \\
89 & 65,44 & 29,67 & 15,67 & 8,03 & 0 & 0 \\
2.222 & 76,10 & 14,15 & 4,45 & 19,57 & 10,19 & 9,55 \\
1.283 & 58,58 & 4,88 & 3,45 & 43,71 & 2,66 & 2,66 \\
2.164 & 98,81 & 5,53 & 0,07 & 64,99 & 5,88 & 5,37 \\
121.963 & 67,01 & 9,76 & 4,81 & 24,99 & 4,69 & 4,38 \\
\hline & & & & & & \\
\hline
\end{tabular}

Legenda: Total - Total de Pacientes Que entraram no Posto no período; Total - Total de Pacientes que saíram do Posto no período; Diárias - Paciente-dia (somatório de pacientes dia a dia); TOH - Taxa de Ocupação Hospitalar; MPe - Média de Permanência; IIS Indice de Intervalo de Substituição; IR - Índice de Renovação; TMGH - Taxa de Mortalidade Geral Hospitalar; TMIn - Taxa de Mortalidade Institucional.

Quadro 11. Outros dados ilustrativos.

\begin{tabular}{|c|}
\hline Cuidados \\
\hline $\begin{array}{ll}\text { - } & \text { Acessos Venosos (AV periférico: 77.590; AV profundo: 32.168) } \\
\text { - } & \text { Medicamento EV específico: } 87.211 \\
\text { - } & \text { Glicemia capilar: } 157.772 \\
\text { - } & \text { Nutrição Parenteral Total: } 161.536 \\
\text { - } & \text { Pressão venosa Central: } 169.341 \\
\text { - } & \text { Dieta Enteral: I 83.064 } \\
\text { - } & \text { Sonda Nasogástrica: } 203.452 \\
\text { - } & \text { Sonda Vesical: (SV Alívio: } 814 ; \text { SV. Volume Urinário: 27.254; SV. Diurese Horária: 867; } \\
\text { - } & \text { SV. Controle Hídrico: } 40.534 \text { ) } \\
\text { - } & \text { Ostonos: 13.738 } \\
\text { - } & \text { Exames com preparo: } 3.405 \\
\text { - } & \text { Números de Curativos: } 69.448 \\
\text { - } & \text { Pré Operatório: } 8.767 \\
\text { - } & \text { Pós Operatório: } 9.367 \\
\text { - } & \text { Cirurgias de Emergência: } 286 \\
\text { - } & \text { Lesões de Pele: } 15.958 \\
\text { - } & \text { Precaução de contato } 25.972 \\
\text { - } & \text { Precaução Respiratória: } 5.127 \\
\text { - } & \text { Prótese Respiratória: } \\
\text { - } & \text { Tubo OrotraQueal: } 5.500 \\
\text { - } & \text { TraQueostomia: } 6.696\end{array}$ \\
\hline
\end{tabular}

Quadro 12. Lista dos Cuidados mais frequentes (SD +SN). 
clientes hospitalizados. Esses cuidados formam uma rede de cuidados cujo padrão de organização e realimentação (feedback) é de uma cadeia continua e diária. Podemos, também, visualizar esses mesmos cuidados nos hospitais públicos e privados credenciados no Brasil, Que são cerca de 5.000 , totalizando cerca de 400 mil leitos e 12 milhões de internação/ano.

O Conhecimento Científico do Cuidado exige capacidade de julgar, explicar, desdobrar, justificar, induzir ou aplicar leis e produzir com segurança eventos futuros transformados em cuidados Que são dados ao cliente adulto enfermo e hospitalizado numa composição em rede. O cuidado recebido por essa clientela, ele é analítico, geral, sistemático, acumulativo, verificáveis e explicativos e observáveis.

\section{REFERÊNCIAS}

I. Certeau M. A invenção do cotidiano: artes de fazer. Petrópolis: Vozes; 1996.

2. Coelho MJ, Figueiredo NMA, Carvalho V. O socorro, o socorrido e o socorrer: Cuidados em Enfermagem de Emergência. Rio de Janeiro: Anna Nery; 1999.

3. Coelho MI. Maneiras de cuidar Enfermagem. Rev Bras Enferm 2006; 59(6): 745-51.
4. Coelho MI. A (in)visibilidade dos cuidados de enfermagem: um ensaio das (re)ações dos cuidados de enfermagem expressos no corpo dos clientes hospitalizados" processo 478920/200 I 6 demanda 05/200 I modalidade APQ. Relatório Final de Pesquisa cuidar/cuidados de Enfermagem Diretório dos Grupos de Pesquisa no Brasil. Rio de Janeiro: Universidade Federal do Rio de Janeiro; 2001. 